\title{
Results from RENO
}

\section{Soo-Bong Kim*}

for the RENO collaboration

Seoul National University, Republic of Korea

E-mail: sbkesnu.ac.kr

The Reactor Experiment for Neutrino Oscillation (RENO) has been taking data near the Hanbit nuclear power plant in South Korea, using two identical detectors since August 2011. The experiment made a definitive measurement of the smallest neutrino mixing angle $\theta_{13}$ in 2012, based on the disappearance of reactor electron antineutrinos. The RENO experiment has obtained more precise values of the mixing angle and the neutrino squared-mass-difference $\left|\Delta m_{e e}^{2}\right|$ from an energy and baseline dependent reactor neutrino disappearance using $\sim 1500$ live days of data. Based on the ratio of inverse-beta-decay (IBD) prompt spectra measured in two identical far and near detectors, we obtain $\sin ^{2}\left(2 \theta_{13}\right)=0.086 \pm 0.006$ (stat. $) \pm 0.005$ (syst.) and $\left|\Delta m_{e e}^{2}\right|=\left[2.61_{-0.16}^{+0.15}(\text { stat. })_{-0.09}^{+0.09}(\right.$ syst. $\left.)\right] \times 10^{-3} e V^{2}$. An excess of reactor antineutrinos near 5 $\mathrm{MeV}$ is observed in the measured prompt spectrum with respect to the most commonly used models. The excess is found to be consistent with coming from reactors and show a weak correlation with the ${ }^{235} \mathrm{U}$ fuel fraction. A precise value of $\theta_{13}$ would provide important information on determination of the leptonic $\mathrm{CP}$ phase if combined with a result of an accelerator neutrino beam experiment.

XVII International Workshop on Neutrino Telescopes

13-17 March 2017

Venezia, Italy

\footnotetext{
* Speaker.
} 


\section{Introduction}

The neutrino oscillation in the framework of three flavors is firmly established when the smallest mixing angle $\theta_{13}$ is finally measured by three reactor neutrino oscillation experiments [1]. It is described by a unitary Pontecorvo-Maki-Nakagawa-Sakata matrix with three mixing angles $\left(\theta_{12}\right.$, $\theta_{23}$ and $\theta_{13}$ ) and one CP phase angle [2]. A rather large value of $\theta_{13}$ opens a new window to determine the $\mathrm{CP}$ violating phase and neutrino mass ordering without a neutrino factory. A next round of neutrino experiments are under consideration or preparation for the determination. A precise measurement of $\theta_{13}$ by a reactor experiment will greatly improve determination of the $\mathrm{CP}$ phase when combined with results of accelerator neutrino beam experiments [3].

Using the $\bar{v}_{e}$ survival probability $P$ [4], reactor experiments with a baseline distance of $\sim 1 \mathrm{~km}$ can determine the mixing angle $\theta_{13}$ and an effective squared-mass-difference $\left|\Delta m_{e e}^{2}\right|$ [5],

$$
\begin{aligned}
1-P= & \sin ^{2} 2 \theta_{13}\left(\cos ^{2} \theta_{12} \sin ^{2} \Delta_{31}+\sin ^{2} \theta_{12} \sin ^{2} \Delta_{32}\right) \\
& +\cos ^{4} \theta_{13} \sin ^{2} 2 \theta_{12} \sin ^{2} \Delta_{21} \\
\approx & \sin ^{2} 2 \theta_{13} \sin ^{2} \Delta_{e e}+\cos ^{4} \theta_{13} \sin ^{2} 2 \theta_{12} \sin ^{2} \Delta_{21},
\end{aligned}
$$

where $\Delta_{i j} \equiv 1.267 \Delta m_{i j}^{2} L / E, E$ is the $\bar{v}_{e}$ energy in $\mathrm{MeV}$, and $L$ is the distance between the reactor and detector in meters. The effective squared-mass-difference is defined by $\Delta m_{e e}^{2} \equiv \cos ^{2} \theta_{12} \Delta m_{31}^{2}+$ $\sin ^{2} \theta_{12} \Delta m_{32}^{2}$, and thus $\left|\Delta m_{e e}^{2}\right|$ is equal to $\left|\Delta m_{32}^{2}\right| \pm \cos ^{2} \theta_{12} \Delta m_{21}^{2}$ where the $+(-)$ sign is for the normal (inverted) mass ordering. Note that $\theta_{13}$ and $\left|\Delta m_{e e}^{2}\right|$ can be unambiguously determined without being affected by the oscillation due to $\theta_{12}$ at the baseline.

The RENO experiment reported an improved measurement of $\theta_{13}$ and the first measurement of $\left|\Delta m_{e e}^{2}\right|$ with a spectral shape and rate analysis using $\sim 500$ days of data [6]. In this talk, we present more precise values of $\theta_{13}$ and $\left|\Delta m_{e e}^{2}\right|$ based on $\sim 1500$ days of data with reduced backgrounds.

\section{The RENO experiment}

The experiment was proposed in 2005, and obtained a full construction fund of $\sim 10 \mathrm{M}$ US dollars in 2006. Civil engineering began in 2007, and both near and far detectors were built in early 2011. Data-taking with both detectors began in August, 2011. As of August 2017, the experiment has collected roughly 2000 live days of data to observe about $2 \mathrm{M}$ reactor neutrino events.

\subsection{Hanbit nuclear reactors}

The Hanbit (known as Yonggwang) nuclear power plant is located in the west coast of southern part of Korea, about $400 \mathrm{~km}$ from Seoul. Six pressurized water reactors, each with maximum thermal output of $2.815 \mathrm{GW}_{t h}$ (reactors 3, 4, 5, and 6) or $2.775 \mathrm{GW}_{t h}$ (reactors 1 and 2), are situated in a line with roughly equal spacings and span a total distance of $\sim 1.3 \mathrm{~km}$ as shown in Fig. 1. A nuclear reactor produces about $10^{20}$ antineutrinos per $\mathrm{GW}_{t h}$, mainly coming from the beta decays of fission products of ${ }^{235} \mathrm{U},{ }^{238} \mathrm{U},{ }^{239} \mathrm{Pu}$, and ${ }^{241} \mathrm{Pu}$. Each fission of the four isotopes releases $\sim 200 \mathrm{MeV}$ energy on average and produces approximately six electron antineutrinos. 


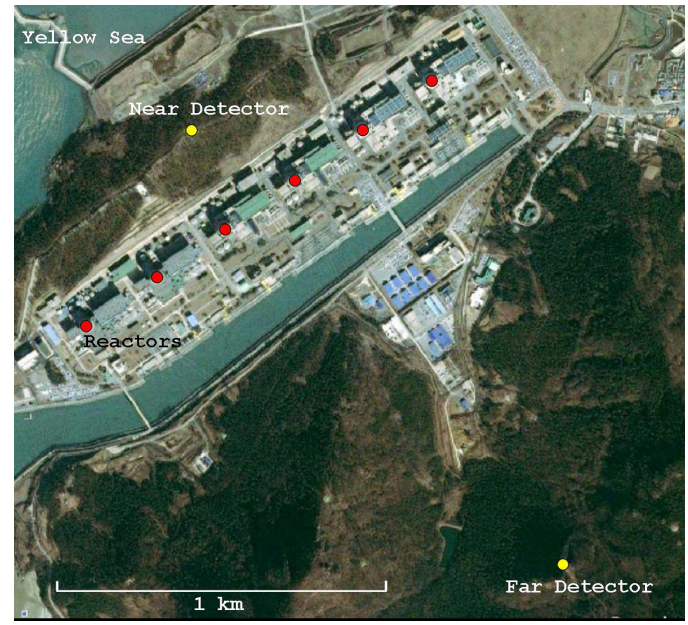

Figure 1: Layout of the RENO experimental site. Red dots and yellow dots represent reactors and detectors, respectively. Six reactors are equally spaced in a $1280 \mathrm{~m}$ span.

\subsection{Two identical detectors}

The identical near and far antineutrino detectors, each having 16.5 tons of Gadolinium (Gd) loaded hydrocarbon liquid scintillator (LS) as a neutrino target, are located at $294 \mathrm{~m}$ and $1383 \mathrm{~m}$, respectively, from the center of the reactor array [7]. A symmetric arrangement of the reactors and the detectors is useful for minimizing the complexity of the measurement. The far (near) detector is under a 450 (120) meters of water equivalent rock overburden. The reactor-flux weighted baseline is $408.56 \mathrm{~m}$ for the near detector, and $1443.99 \mathrm{~m}$ for the far detector. The positions of two detectors and six reactors are surveyed with GPS and total station to determine the baseline distances between the detectors and reactors to an accuracy of better than $10 \mathrm{~cm}$. Reactor neutrino fluxes at the two detectors are obtained by calculating the reduction effect of the baseline distances to a precision of much better than $0.1 \%$. A measured near-to-far ratio of antineutrinos can considerably reduce several systematic errors coming from uncertainties in the reactor neutrino flux, target mass, and detection efficiency [8]. The relative measurement is independent of near-far correlated uncertainties and helps in minimizing uncorrelated reactor uncertainties [9].

\subsection{Data-taking status}

RENO was the first reactor experiment to take data with two identical near and far detectors in operation, from August 2011. In early April 2012, the experiment successfully reported a definitive measurement of $\theta_{13}$ by observing the disappearance of reactor neutrinos [1]. As of August 2017, the experiment has collected roughly 2000 live days of data with data-taking efficiency of better than $95 \%$.

\section{The RENO detector}

Each RENO detector, having a cylindrical shape of $8.8 \mathrm{~m}$ in height and $8.4 \mathrm{~m}$ in diameter, consists of a main inner detector (ID) and an outer veto detector (OD). The ID is contained in a 
cylindrical stainless steel vessel of $5.4 \mathrm{~m}$ in diameter and $5.8 \mathrm{~m}$ in height which houses two nested cylindrical acrylic vessels [7]. The acrylic vessels holding organic liquids are made of casted polymethylmethacrylate (PMMA) plastic which transmits up to $92 \%$ of visible light at $3 \mathrm{~mm}$ thickness and reflects about $4 \%$ from the surfaces [10]. Both near and far detectors are built as identical as possible. For example, the acrylic target vessels of the two detectors are compared for volume, using water, before installation, and the volume difference is found to be $2.5 \ell$ corresponding to $0.02 \%$ of the total volume. A total 354 of low background 10-inch Hamamatsu R7081 PMTs [11] are mounted in a uniform array and on the inner surface of a cylindrical stainless steel vessel, providing $14 \%$ photo-sensitive surface coverage. The vessel optically isolates the inner detector from the outer veto. The applied high voltages to the PMTs are monitored and controlled in real time by a slow control system [12]. The RENO LS is developed and produced as a mixture of linear alkylbenzene (LAB), $3 \mathrm{~g} / \ell$ of PPO, and $30 \mathrm{mg} / \ell$ of bis-MSB [13]. A Gd-carboxylate complex using trymethylhexanoid acid (TMHA) was developed for the best Gd loading efficiency into LS and its long term stability. The LS loaded with Gd was equally divided to be filled into the detectors for every batch of production. This minimized the difference of Gd concentration between near and far detectors.
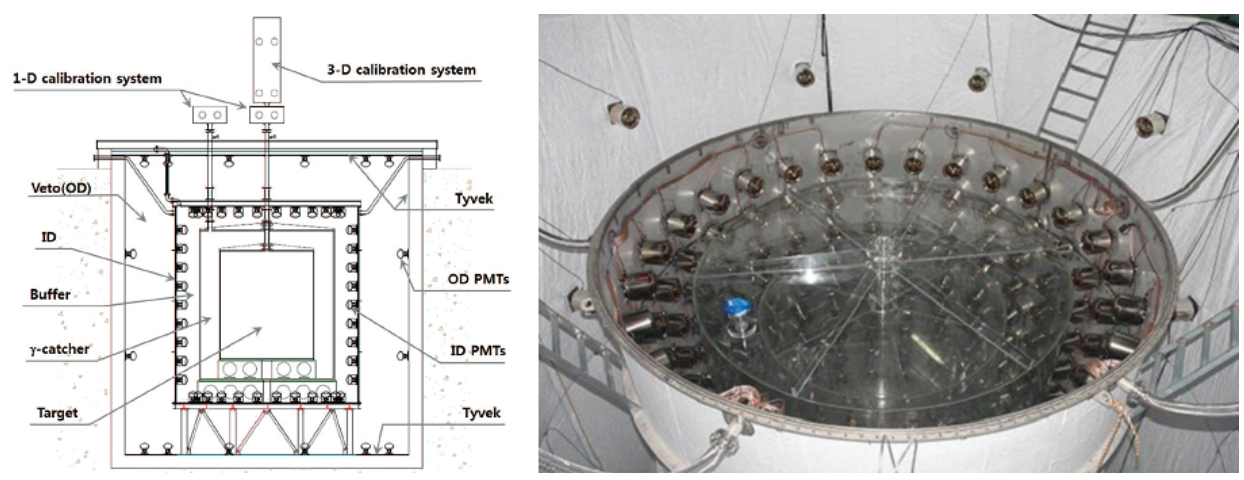

Figure 2: A schematic view of RENO detector (left) and a photo of the detector interior with PMTs installed (right). The ID is contained in a cylindrical stainless steel vessel of $5.4 \mathrm{~m}$ in diameter and $5.8 \mathrm{~m}$ in height, and the OD is surrounded by a cylindrical concrete vessel of $8.8 \mathrm{~m}$ in height and $8.4 \mathrm{~m}$ in diameter. The ID is viewed by 35410 -inch PMTs that are mounted on the inner wall of the stainless steel container. The OD is equipped with 67 10-inch PMTs mounted on the wall of the concrete vessel.

\section{Energy calibration}

The reactor $\bar{v}_{e}$ is detected through the inverse beta decay (IBD) interaction, $\bar{v}_{e}+p \rightarrow e^{+}+n$, with free protons in LS with $0.1 \% \mathrm{Gd}$ as a target. The coincidence of a prompt positron signal and a $\sim 28 \mu$ s delayed signal from neutron capture by Gd (n-Gd) provides the distinctive IBD signature against backgrounds. The prompt signal releases energy of $1.02 \mathrm{MeV}$ as two $\gamma$-rays from the positron annihilation in addition to the positron kinetic energy. The delayed signal produces several $\gamma$-rays with the total energy of $\sim 8 \mathrm{MeV}$. In the IBD reaction, the energy of the incident $\bar{v}_{e}$ is given by

$$
E_{\bar{v}_{e}}=E_{e^{+}}+T_{n}+\left(m_{n}-m_{p}\right) \approx E_{e^{+}}+1.293 \mathrm{MeV}
$$


where $E_{e^{+}}$is the total energy of the positron, $m_{n}\left(m_{p}\right)$ is the neutron (proton) mass, and $T_{n}$ is the negligibly small kinetic energy of the recoil neutron.

Event triggers are based on the number of hit PMTs $\left(N_{h i t}\right)$ with signals above a $\sim 0.3$ photoelectron (p.e.) threshold. An event passes trigger selection and is recorded if the ID $N_{h i t}$ is larger than 90 , corresponding to $0.5-0.6 \mathrm{MeV}$ and well below the $1.02 \mathrm{MeV}$ minimum energy of an IBD positron signal. The visible event energy is determined from the total charge $\left(Q_{t o t}\right)$ in p.e. that is collected by the PMTs within $-100 \mathrm{~ns}$ to $+50 \mathrm{~ns}$ and corrected for gain and charge collection variations using the neutron capture peak energies.

An absolute energy measurement is essential for measuring $\left|\Delta m_{e e}^{2}\right|$ and $\theta_{13}$ from the spectral distortion of IBD prompt events that is developed by neutrino oscillation. We used commercially available radioactive sources of ${ }^{137} \mathrm{Cs},{ }^{68} \mathrm{Ge},{ }^{60} \mathrm{Co},{ }^{210} \mathrm{Po}{ }^{9} \mathrm{Be}$ and ${ }^{252} \mathrm{Cf}$ to calibrate the absolute energy scale. A charge-to-energy conversion function is generated from the peak energies of the $\gamma$-ray sources. The observed $Q_{t o t}$ of a $\gamma$-ray source is converted to the corresponding $Q_{t o t}$ of a positron $\left(Q_{t o t}^{c}\right)$ using a GEANT4 Monte Carlo simulation. The true visible energy $\left(E_{\text {true }}\right)$ of a positron is the sum of the kinetic energy and the energy from its annihilation. The converted $Q_{t o t}^{c}$ of IBD prompt energy $\left(E_{p}\right)$ is estimated by taking into account difference in the visible energies of a $\gamma$-ray and a positron through the MC. The RENO MC includes measured optical properties of LS and quenching effect of $\gamma$-ray at low energies [13]. The quenching effect depends on the energy and the multiplicity of $\gamma$-rays released from the calibration sources. The MC simulated $Q_{t o t}$ well reproduces that of a $\gamma$-ray source including the quenching effect depending on the multiplicity of $\gamma$-rays. The measured $Q_{t o t}^{c}$ shows non-linear response to $E_{\text {true }}$, especially at low energies, mainly due to quenching effect in the scintillator and Cherenkov radiation. Figure 3 upper panel shows non-linear response of scintillating energy for the IBD prompt signal which is well described by a parametrization of $Q_{t o t} / E_{\text {true }}=a+b /\left[1-\exp \left(-c E_{\text {true }}+d\right)\right]$. The parameters $a, b, c$, and $d$ are determined by a fit. Deviation of all calibration data points with respect to the best-fit is within $1 \%$ as shown in Fig. 3 lower panel. The energy scales of the near and far detectors are compared using identical radioactive sources, and the difference is found to be less than $0.15 \%$ for $E_{p}=1-8 \mathrm{MeV}$

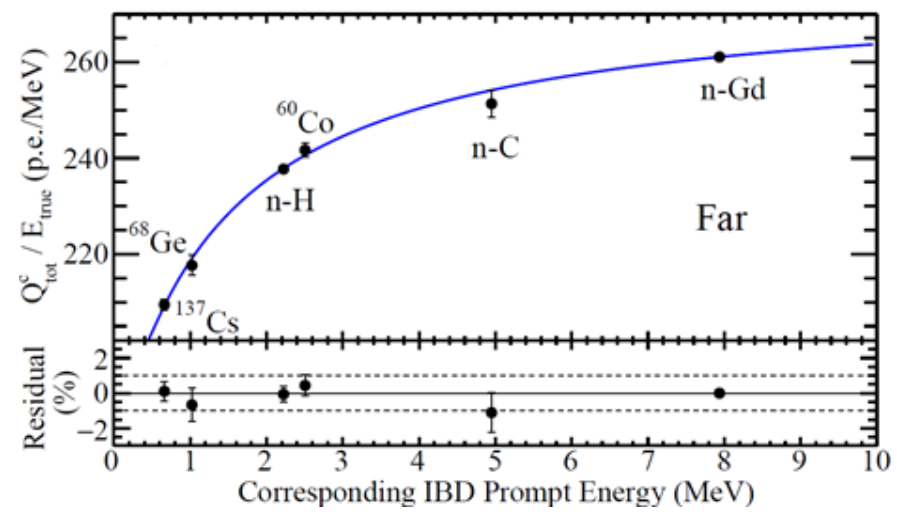

Figure 3: Non-linear response of scintillating energy obtained from the visible energies of $\gamma$-rays coming from several radioactive sources and IBD delayed signals in the far detector. The curve is the best fit to the data points. Note that the $\mathrm{n}-\mathrm{C}$ sample is obtained from the ${ }^{209} \mathrm{Po}^{9} \mathrm{Be}$ source. 


\section{Data sample and event selection}

RENO has collected roughly 2000 live days of data as of August 2017 to observe $\sim 1.8 \mathrm{M}$ reactor neutrino events in the near detector and $\sim 0.2 \mathrm{M}$ events in the far detector. We have analyzed 1500 days of data in the period between August 2011 and September 2015, to obtain spectral measurement results on $\theta_{13}$ and $\left|\Delta m_{e e}^{2}\right|$. Because of different overburdens the near and far detectors suffer from unequal cosmogenic-background rates and uncertainties.

The IBD selection criteria are modified after the previous RENO measurement [6] to reduce background rates and uncertainties. A set of newly optimized timing veto criteria are applied to reject additional ${ }^{9} \mathrm{Li} /{ }^{8} \mathrm{He}$ background events associated with the high-energy cosmic muons if they are within a $1 \mathrm{~s}(800 \mathrm{~ms}, 500 \mathrm{~ms}, 100 \mathrm{~ms})$ window following a cosmic muon of the visible energy $E_{\mu}>1.5 \mathrm{GeV}(1.3-1.5 \mathrm{GeV}, 1.1-1.3 \mathrm{GeV}, 0.85-1.1 \mathrm{GeV})$ for the far detector, or within a 800 ms $(300 \mathrm{~ms}, 200 \mathrm{~ms}, 50 \mathrm{~ms})$ window following a cosmic muon of the visible energy $E_{\mu}>1.6$ $\mathrm{GeV}(1.4-1.6 \mathrm{GeV}, 1.3-1.4 \mathrm{GeV}, 1.1-1.3 \mathrm{GeV})$ for the near detector. The remaining ${ }^{9} \mathrm{Li} /{ }^{8} \mathrm{He}$ background is reduced by $40.0 \%$ (34.4\%) in the far (near) detector. A tighter spatial correlation of $\Delta R<2.0 \mathrm{~m}$ and an improved PMT flasher removal criterion are imposed to reduce the accidental background rate by $60.6 \%(69.9 \%)$ for the far (near) detector. The accidental background uncertainty is reduced from $3.1 \%(1.2 \%)$ to $2.3 \%$ (0.9\%) for the far (near) detector. This reduction has resulted in significant reduction of the systematic error for the $\left|\Delta m_{e e}^{2}\right|$. A tighter timing and spatial veto requirement is applied to reduce the ${ }^{252} \mathrm{Cf}$ contamination background. In the previous measurement published in Ref. [6] the background events are rejected if they are accompanied by a prompt candidate of $E_{p}>3 \mathrm{MeV}$ within a $10 \mathrm{~s}$ window and a distance of $40 \mathrm{~cm}$. Additional background events are removed by extending the timing veto window to $20 \mathrm{~s}$ and the spatial distance to $50 \mathrm{~cm}$.

Table 1: Event rates of the observed IBD candidates and the estimated background at $1.2<E_{p}<8.0 \mathrm{MeV}$. Rates are given in per day.

\begin{tabular}{lrr}
\hline \hline Detector & Near & Far \\
\hline Number of selected events & 732168 & 68055 \\
Total background rate & $9.34 \pm 0.37$ & $1.95 \pm 0.15$ \\
IBD rate after background subtraction & $463.80 \pm 0.66$ & $46.75 \pm 0.24$ \\
DAQ live time (days) & 1547.35 & 1397.78 \\
\hline Accidental rate & $2.07 \pm 0.02$ & $0.38 \pm 0.01$ \\
${ }^{9} \mathrm{Li} /{ }^{8} \mathrm{He}$ rate & $5.49 \pm 0.36$ & $0.93 \pm 0.15$ \\
Fast neutron rate & $1.74 \pm 0.02$ & $0.35 \pm 0.01$ \\
${ }^{252} \mathrm{Cf}$ contamination rate & $0.04 \pm 0.01$ & $0.28 \pm 0.02$ \\
\hline \hline
\end{tabular}

Applying the IBD selection criteria yields 68055 (732168) candidate events with $E_{p}$ between 1.2 and $8.0 \mathrm{MeV}$ for a live time of 1397.78 (1547.35) days in the far (near) detector. IBD events with $E_{p}<1.2 \mathrm{MeV}$ include prompt signals of positrons occurring in or near the target acrylic vessel that deposit kinetic energy in the acrylic without producing scintillation lights. These events are reconstructed to have visible energy near the positron annihilation energy of $1.02 \mathrm{MeV}$ and are 
not well reproduced by the MC prediction. The IBD signal loss by $E_{p}<1.2 \mathrm{MeV}$ requirement is roughly $2 \%$ in both detectors. The total background rates are estimated to be $9.34 \pm 0.37$ and $1.95 \pm 0.15$ events per day for near and far detectors, respectively. The observed IBD and background rates are summarized in Table 1.

Table 2: Systematic uncertainties in the reactor neutrino detection. The systematic uncertainties of the detection efficiency are correlated or uncorrelated between the near and far detectors. The systematic uncertainties used in the reactor neutrino flux estimation are correlated or uncorrelated among reactors.

\begin{tabular}{lrr}
\hline \hline \multicolumn{2}{c}{ Uncorrelated } & Correlated \\
\hline Thermal power & Reactor & - \\
Fission fraction & $0.5 \%$ & - \\
Fission reaction cross section & $0.7 \%$ & $1.9 \%$ \\
Reference energy spectra & - & $0.5 \%$ \\
Energy per fission & - & $0.2 \%$ \\
\hline Combined & - & $2.0 \%$ \\
\hline \hline & $0.9 \%$ & $0.13 \%$ \\
\hline IBD cross section & & $0.10 \%$ \\
Target protons & - & $0.09 \%$ \\
Prompt energy cut & $0.03 \%$ & $0.01 \%$ \\
$Q_{\text {max }} / Q_{t o t}$, flasher cuts & $0.01 \%$ & $0.47 \%$ \\
Gd capture ratio & $0.02 \%$ & $0.50 \%$ \\
Delayed energy cut & $0.10 \%$ & $0.45 \%$ \\
Time coincidence cut & $0.05 \%$ & $0.02 \%$ \\
Spatial correlation cut & $0.01 \%$ & $0.61 \%$ \\
Spill-in & $0.02 \%$ & $1.04 \%$ \\
\hline Combined (total) & $0.04 \%$ & \\
\hline \hline
\end{tabular}

\section{Systematic uncertainties}

Uncorrelated relative uncertainties are estimated by comparing the two identical detectors. The uncertainty of the IBD differential cross section [14] is correlated and canceled out. The GdLS was commonly produced, divided equally and filled into the two detectors for their identical Gd concentration and target proton numbers. This results in a difference in the number of the target protons less than $0.1 \%$. The difference in the measured neutron capture time between the detectors is less than $0.2 \mu \mathrm{s}$, corresponding to Gd concentration differences of less than $0.1 \%$. The relative uncertainty of Gd capture ratio is less than $0.1 \%$ accordingly. The energy scales of the near and far detectors are compared using identical radioactive sources, and the difference is less than $0.15 \%$ for $E_{p}=1-8 \mathrm{MeV}$. The energy scale difference corresponds to a relative uncertainty of $0.05 \%$ in the delayed energy cut efficiency. The remaining relative detection efficiency uncertainties are close to 
$0.01 \%$, and the combined uncorrelated uncertainty is $0.13 \%$ while the total correlated uncertainty of detection efficiency is $1.04 \%$ [6].

The absolute uncertainties of the efficiencies are mostly correlated between the two detectors. Only differences between the two identical detectors are taken as uncorrelated uncertainties. The systematic uncertainties are summarized in Table 2. The background uncertainties of $4.0 \%$ for the near detector and $7.7 \%$ for the far detector are assumed to have no correlation. The uncorrelated uncertainties among reactors are found to be $0.5 \%$ per core due to the thermal power uncertainty and $0.7 \%$ due to the fission fraction uncertainty as listed in Table 2. The energy dependent systematic uncertainties coming from background shape ambiguities are evaluated and included for this analysis.

\section{The $5 \mathrm{MeV}$ excess}

The expected rates and spectra of reactor antineutrinos are calculated for duration of physics data-taking, taking into account the varying thermal powers and fission fractions. RENO has obtained an unprecedentedly accurate measurement of the reactor antineutrino flux and spectrum. Figure 4 shows a spectral comparison of the background subtracted IBD prompt spectrum to the prediction that is expected from a reactor neutrino model [15] and the best fit oscillation results. The subtracted background spectra are shown in the insets. A clear spectral difference is observed in the region centered at $5 \mathrm{MeV}$. This was reported in Ref. [6] and in the conference of Neutrino 2014 [16]. The MC predicted distributions are normalized to the observed events excluding the excess range $3.6 \mathrm{MeV}<E_{p}<6.6 \mathrm{MeV}$. The excess of events constitutes about $2.5 \%$ of the total observed reactor $\bar{v}_{e}$ rate in both detectors. Furthermore, the excess is observed to be proportional to the reactor power, indicating that the excess of IBD events comes from the reactors. This observation suggests needs for reevaluation and modification of the current reactor $\bar{v}_{e}$ model [17].
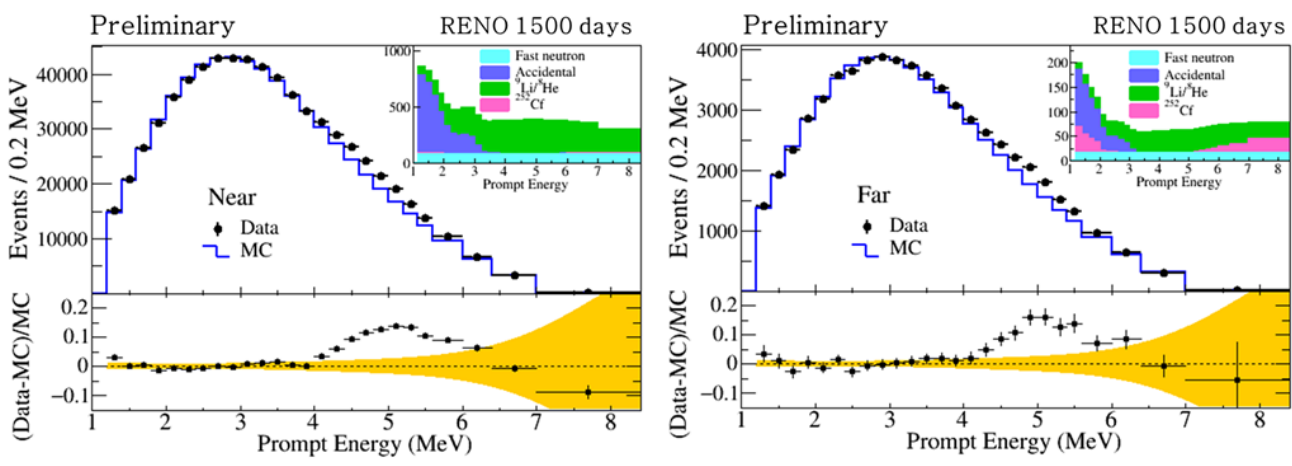

Figure 4: The observed spectra are obtained from subtracting the background spectra as shown in the insets. A shape difference is clearly seen at $5 \mathrm{MeV}$. A spectral deviation from the expectation is larger than the uncertainty of an expected spectrum (shaded band).

We have studied to find a possible correlation of the $5 \mathrm{MeV}$ excess with respect to any of the fuel isotopes using the near IBD data sample. The ${ }^{235} \mathrm{U}$ fraction indicates the fuel freshness, and 
thus is the largest at the beginning of reactor fuel cycle. Figure 5 shows the fractional magnitude of the $5 \mathrm{MeV}$ excess relative to the observed reactor $\bar{v}_{e}$ rate as a function of the average ${ }^{235} \mathrm{U}$ fraction that is weighted by the multiple reactor thermal powers. Within the statistical uncertainty there seems to be a weak correlation between them at present.

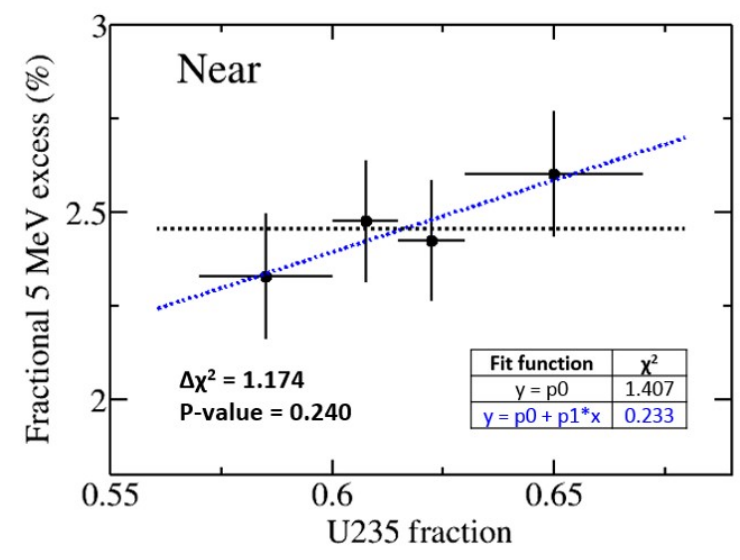

Figure 5: Correlation of the $5 \mathrm{MeV}$ excess observed in the near detector with respect to the ${ }^{235} \mathrm{U}$ fraction on average over the multiple reactor thermal powers. It shows a weak correlation between them according to the calculated $\Delta \chi^{2}$ value.

\section{Spectral measurement of $\theta_{13}$ and $\left|\Delta m_{e e}^{2}\right|$}

The RENO's first measurement result of $\left|\Delta m_{e e}^{2}\right|$ was obtained from the 500 day data sample, and was published in Ref. [6]. Here we report a more precise spectral-measurement of $\left|\Delta m_{e e}^{2}\right|$ and $\theta_{13}$. Because of the unexpected structure around $5 \mathrm{MeV}$, the oscillation amplitude and frequency are determined from a fit to the measured far-to-near ratio of IBD prompt spectra. The relative measurement using identical near and far detectors makes the method insensitive to the correlated uncertainties of expected reactor $\bar{v}_{e}$ flux and spectrum as well as detection efficiency. To determine $\left|\Delta m_{e e}^{2}\right|$ and $\theta_{13}$ simultaneously, a $\chi^{2}$ is constructed using the spectral ratio measurement and is minimized with respect to the pull parameters and the oscillation parameters. [9].

The best-fit values obtained from the rate and spectral analysis are $\sin ^{2}\left(2 \theta_{13}\right)=0.086 \pm$ 0.006 (stat.) \pm 0.005 (syst.) and $\left|\Delta m_{e e}^{2}\right|=\left[2.61_{-0.16}^{+0.15}(\text { stat. })_{-0.09}^{+0.09}(\right.$ syst. $\left.)\right] \times 10^{-3} \mathrm{eV}^{2}$ with $\chi^{2} / N D F=$ $59.9 / 66$. The dominant systematic uncertainties are those of the energy scale difference and the backgrounds.

Figure 6 shows the background-subtracted, observed spectrum at far detector compared to one expected for no oscillation and the other expected for the best-fit oscillation at the far detector. The expected spectra are obtained by weighting the spectrum at near detector with the oscillation or no oscillation assumptions using the measured values of of $\theta_{13}$ and $\left|\Delta m_{e e}^{2}\right|$. The observed spectrum shows a clear energy-dependent disappearance of reactor $\bar{v}_{e}$ consistent with neutrino oscillations. Because of the relative measurement using the measured far-to-near ratio of IBD prompt spectra, the unexpected spectral structure around $5 \mathrm{MeV}$ does not affect the measured values of $\theta_{13}$ and $\left|\Delta m_{e e}^{2}\right|$ by the spectral analysis. 


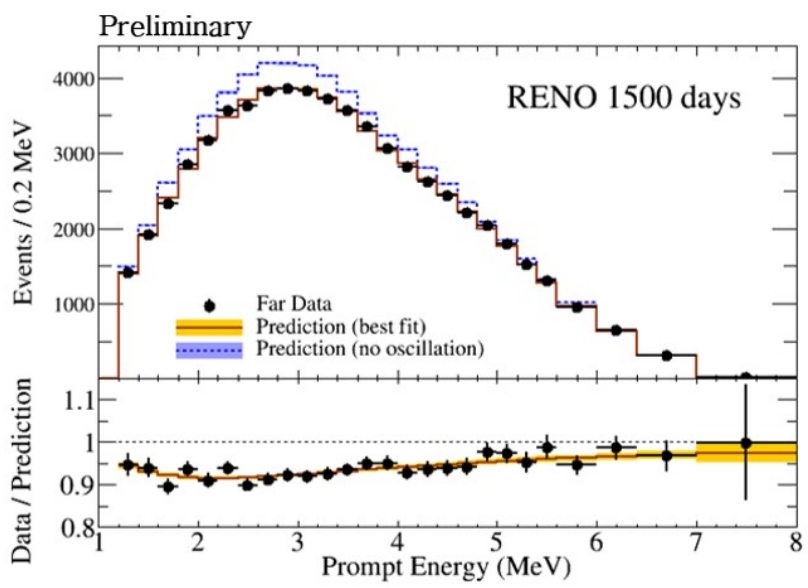

Figure 6: Top: comparison of the observed IBD prompt spectrum in the far detector with the no-oscillation prediction obtained from the measurement in the near detector. The prediction from the best-fit results to oscillation is also shown. Bottom: ratio of reactor $\bar{v}_{e}$ events measured in the far detector to the no-oscillation prediction (points) and ratio from MC with best-fit results folded in (shaded band).

Figure 7 shows the measured survival probability of reactor $\bar{v}_{e}$ as a function of an effective baseline $L_{e f f}$ over $\bar{v}_{e}$ energy $E_{v}$ in the far detector, in a good agreement with the prediction that is obtained from the observed distribution in the near detector, for the best-fit oscillation values. This result demonstrates clear $L_{e f f} / E_{v}$-dependent disappearance of reactor $\bar{v}_{e}$, consistent with the periodic feature of neutrino oscillation. Note that $L_{e f f}$ is the reactor-detector distance weighted by the multiple reactor fluxes, and $E_{v}$ is converted from the IBD prompt energy. The measured survival probability is obtained by the ratio of the observed IBD counts to the expected counts assuming no oscillation in each bin of $L_{e f f} / E_{v}$.

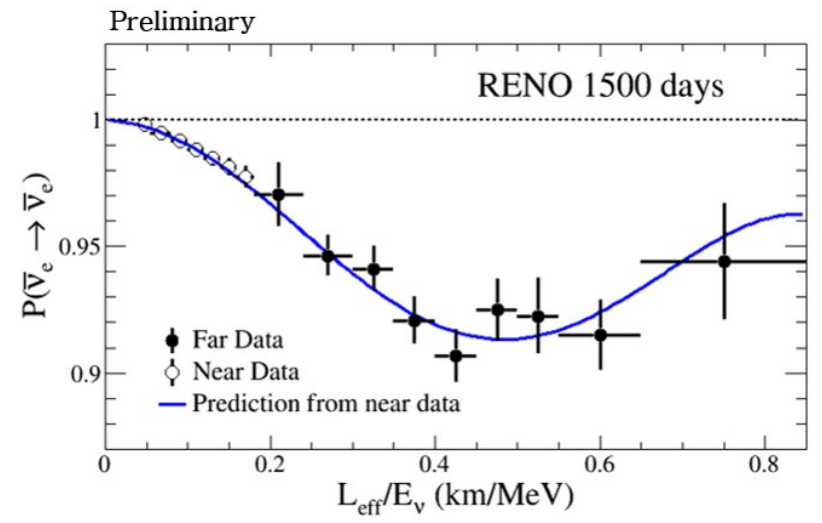

Figure 7: Measured reactor $\bar{v}_{e}$ survival probability in the far detector as a function of $L_{e f f} / E_{v}$. The curve is a predicted survival probability, obtained from the observed probability in the near detector, for the best-fit values of $\left|\Delta m_{e e}^{2}\right|$ and $\theta_{13}$. The $L_{e f f} / E_{v}$ value of each data point is given by the average of the counts in each bin. 


\section{Acknowledgments}

The RENO experiment is supported by the National Research Foundation of Korea (NRF) grant No. 2009-0083526 funded by the Korea Ministry of Science and ICT. Some of us have been supported by a fund from the BK21 of NRF. We gratefully acknowledge the cooperation of the Hanbit Nuclear Power Site and the Korea Hydro \& Nuclear Power Co., Ltd. (KHNP). We thank KISTI for providing computing and network resources through GSDC, and all the technical and administrative people who greatly helped in making this experiment possible.

\section{References}

[1] Y. Abe et al. (Double Chooz Collaboration), Phys. Rev. Lett. 108 (2012) 131801; F.P. An et al. (Daya Bay Collaboration), Phys. Rev. Lett. 108 (2012) 171803; J.K. Ahn et al. (RENO Collaboration), Phys. Rev. Lett. 108 (2012) 191802.

[2] B. Pontecorvo, Sov. Phys. JETP 7 (1958) 172; Z. Maki, M. Nakagawa, and S. Sakata, Prog. Theor. Phys. 28 (1962) 870.

[3] K. Abe et al. (T2K Collaboration), Phys. Rev. Lett. 112 (2014) 061802; J. Bian et al. (NOnA Collaboration), [hep-ex/1510.05708] (2015).

[4] S. T. Petcov and M. Piai,Phys. Lett. B 533 (2002) 94.

[5] H. Nunokawa, S. Parke, and R. Zukanovich Funchal, Phys. Rev. D 72, 013009 (2005) 013009; S. Parke, Phys. Rev. D 93 (2016) 053008.

[6] J.H. Choi et al. (RENO Collaboration), Phys. Rev. Lett. 116 (2016) 211801; S.H. Seo et al. (RENO Collaboration), [hep-ex/1610.04326] (2016).

[7] J.K. Ahn et al. (RENO Collaboration), RENO Proposal and TDR [hep-ex/1003.1391] (2010).

[8] L. A. Mikaelyan and V. V. Sinev, Phys. Atomic Nucl. 63 (2000) 1002.

[9] K. Anderson et al., White paper report on using nuclear reactors to search for a value of $\theta 13$, [hep-ex/0402041] (2004).

[10] K.S. Park et al. (RENO Collaboration), Nucl. Instrum. Meth. A 686 (2012) 91-99.

[11] K.J. Ma et al. (RENO Collaboration), Nucl. Instrum. Meth. A 629 (2011) 93-100.

[12] J.H. Choi et al. (RENO Collaboration), Nucl. Instrum. Meth. A 810 (2016) 100-106.

[13] J.S. Park et al. (RENO Collaboration), Nucl. Instrum. Meth. A 707 (2013) 45-53.

[14] P. Vogel and J.F. Beacom, Phys. Rev. D 60 (1999) 053003.

[15] F. von Feilitzsch, and K. Schreckenbach, Phys. Lett. B118 (1982) 162; W.G.K. Schreckenbach, G. Colvin, and F. von Feilitzsch, Phys. Lett. B160 (1985) 325; A.A. Hahn et al., Phys. Lett. B218 (1989) 365.

[16] See a talk given by Seon-Hee Seo at 26th International Conference on Neutrino Physics and Astrophysics, Boston, USA, 2-7 June, 2014.

[17] Th. A. Mueller et al., Phys. Rev. C 83 (2011) 054615; P. Huber, Phys. Rev. C 84 (2011) 024617; 85 (2012) 029901(E). 